\title{
Toward a Data-Driven Performance-Guided Urban Decision-Support Environment
}

\author{
Neda Ghiassi, Stefan Glawischnig, Ulrich Pont, and Ardeshir Mahdavi \\ Vienna University of Technology, \\ Department of Building Physics and Human Ecology Vienna, Austria \\ \{neda.ghiassi, stefan.glawischnig, ulrich.pont, \\ amahdavi\}@tuwien.ac.at
}

\begin{abstract}
The present contribution briefly represents the structure and main features of SEMERGY, a performance-guided multi-objective building optimization environment, supported by Semantic Web technologies. It establishes the importance of urban-scale performance considerations, discusses particular features of urban data, and suggests a framework to upscale the SEMERGY approach towards development of a data-driven performance-guided urban decision support environment. The suggested task-based ontology framework can facilitate data and knowledge sharing within the domain of urban performance inquiries.
\end{abstract}

Keywords: GIS. Semantic Web Technologies. Ontologies. Data-Driven. Building Performance. Urban Performance. Urban planning. Sustainability. Information Systems.

\section{Introduction}

Building Performance Evaluation (BPE), defined as "the process of obtaining knowledge about building performance and using the feedback to improve buildings" originated in the 1960's [1]. Ever since, efforts have been directed towards development and refinement of tools and methods to assess and/or predict the performance of buildings in terms of use of natural resources, contribution to undesired environmental phenomena, and user comfort.

Despite the advances in the development of Building Performance Evaluation (BPE) tools over the past decades, the adoption of such tools to support and guide design decisions has been relatively slow and their implementation mostly limited to certification purposes [2, 3]. Among stated reasons for the insufficient adoption of such tools for sustainable planning and informed decision making were non-technical, socio-cultural barriers such as lack of financial justification, lack of motivation, or professional skepticism and prejudices [3, 4]. Stated technical barriers include the discordance of the available tools with the capabilities and expectations of the design community [5] and complexities related to data availability and accessibility, which render the process of data provision for BPE applications cumbersome, error prone and time consuming [6]. 
The SEMERGY project [7, 8], was a recent (2011-2013) research and development effort, intended to address the data-related technical barriers to the integration of performance assessment tools in the building design process. SEMERGY aimed to explore the potential of semantic web technologies to facilitate the process of data accumulation and entry for building performance evaluation and optimization.

Although integration of performance evaluation in the design of new buildings and retrofit projects supports informed decisions and sustainable design, an approach focused on individual buildings may not be singularly sufficient to insure energy efficiency and sustainability of the built environment. Certain performance aspects of the built environment, such as microclimatic conditions (air flows, incident solar radiation) or energy flows are only properly grasped, when buildings are regarded as active agents within a network of interconnected and inter-influential elements, as opposed to independent entities isolated from the context. Such a perspective reveals opportunities and issues, which may have been overlooked, when considering a single building.

On the other hand, the decision to improve the performance of a building depends mainly on the owner, who may be affected by the previously-stated barriers such as lack of motivation or financial means for such an undertaking. As such performance guided decision making has to be endorsed also at a higher level by local/national authorities to support conception of sustainability strategies, efficient allocation of resources and identification of opportunities. Intended as a comprehensive software environment to facilitate performance-guided decision making at various scales, this top-down perspective was also included in the initial description of the SEMERGY project as a future use-case. As such, a second phase of the SEMERGY project has been planned to support performance-enhancing decisions transcending the scale of a single building, through utilization of Semantic Web technologies to harness the potential of the immense pool of web-based urban data.

The present contribution briefly represents the structure and main features of the SEMERGY environment, discusses the particularities of urban scale performance considerations, offers an overview of related previous research, and suggests a framework to upscale the SEMERGY approach towards development of a data-driven performance-guided urban decision support environment.

\section{SEMERGY Structure and Features}

As mentioned earlier, the primary objective of the SEMERGY project was to help remove data-related disincentives to integration of BPE in building design process. SEMERGY intended to explore developmental opportunities toward effective evaluation environments for comparative assessment of alternative design and retrofit options [7], focusing in particular (as proof of concept) on the scattered pool of webbased building product and material data. 
The overall structure of SEMERGY is depicted by Figure 1. SEMERGY consists of three main components: The User Interface, which is reponsible for the communication of user intents and initial design to the program, the Reasoning Interface, which includes the relevant performance computation methods and the optimization algorithms, and most importantly, the Semantic Interface which provides links between SEMERGY and web-based data sources to facilitate semi-automatic extraction of appropriate data.

Efficient generation of BPE-compliant models is hampered in part by the missing link between users' simplified component representations (e.g., "external wall", "window") versus the complexity of specifications of real world products. In other words, users are obliged to map such simple notions of building components to appropriate real world products that meet calculation procedures' informational requirements [8]. In optimization tasks, the model iteratively assumes alternative sets of properties and is subjected to the evaluation procedures, which lead to the identification of the optimal set of solutions according to the estimated values of functional, ecological, and economical performance indicators.

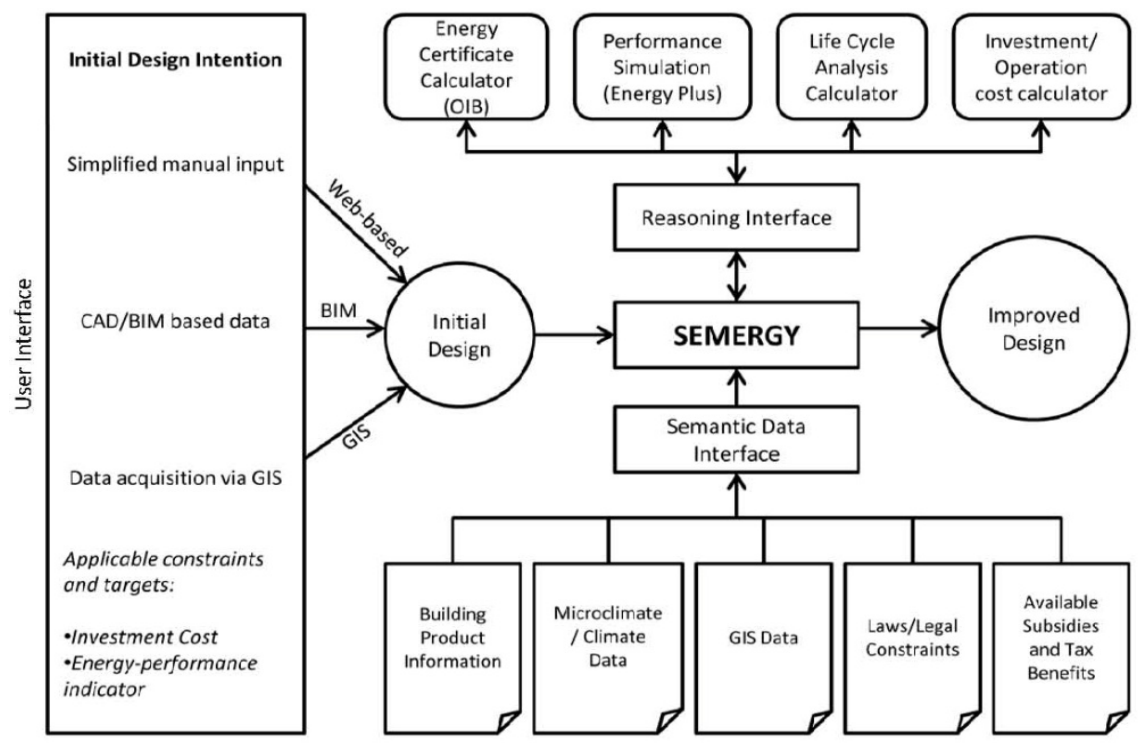

Fig. 1. SEMERGY structure

The SEMERGY project's approach to bridge the gap between required and available AEC data sets was based on two main pillars: First, creation of a compact and versatile ontology of building products compliant with a shared perception of AEC concepts; Second, mapping of the scattered building product information resources on the web to this ontology [8].

To achieve an acceptable building data representation, compatible with the requirements of BPE methods of various resolutions and precisions, and in accordance 
with the notion of the design community, various building data models were studied and several BPE applications were reversed analyzed. These efforts led to the development of a coherent and sufficient view of building data, known as the SEMERGY Building Model (SBM) [9]. Further on, an ontology of building products and construction materials was created, which structurally complied with the developed model. This ontology was linked to data sources, which represent building products by their various physical attributes, production data and cost information. The property inheritance structure of the ontology allowed the systematic enrichment of the extracted data with additional properties, which are obvious to human observer (such as stability of shape), yet essential for rule based queries that support the semi-automatic identification of design alternatives, in accordance with user-specified preferences and constraints $[10,11,12,13]$. The same method can theoretically be applied to other relevant types of web-based data such as building codes, financial subsidies, microclimatic data, building systems, etc. Since these domain ontologies are developed based on a unique perception of a building (SBM) or mapped to it, they can be combined to cater for various types of inter-domain inquiries. For instance, building systems data and building product data can be used in combination to identify the optimal retrofit strategies for a historical building, according to budget limitations expressed by the user.

This approach is expected to encourage the utilization of BPE methods to guide design and retrofit of individual buildings by overcoming certain structural barriers to data accumulation and entry. However, data availability and accuracy issues still hamper the systematic implementation of such a method due to the wide distribution of data sources and lack of standard data formats acknowledged by various data providers.

\section{Particularities of Urban Data}

\subsection{Data size}

Decision making and development of policies and strategies at an urban scale require dependable, robust, and up-to-date information. A large amount of potentially useful data exists on-line. Much of this data is either partially available or is intended to be made available to the public as part of open data initiatives (e.g., demographics, detailed representations of buildings and their various properties, transportation patterns, energy flows, natural resources, etc.). Analyzing this large source of rather unstructured data with conventional methods may not lead to the discovery of opportunities, detection of systemic problems or leakages of resources.

\subsection{Data Diversity}

City is an ecosystem composed of intertwined and interacting sub-systems, which may be physical or virtual, static or dynamic, predictable or complex, alive or inanimate. Buildings, climate and natural context, transport systems, media, energy production and distribution networks, financial and legal structures, inhabitants, etc. are 
all various subsystems forming this ecosystem. The entire system's performance is a product of the various chains of events within different sub-systems and the interactions between them. Data pertaining to these various sub-systems is collected and organized by domain specialists from different sectors. The availability of data on various aspects of the city presents an immense potential for conception of interdomain sustainability strategies. Nonetheless, due to this diversity, navigation through the available data - to identify data sources relevant to a certain inquiry - may not be trivial.

\subsection{Multiple Perceptions, Multiple Representations}

Urban data is produced and accumulated by various stakeholders, each perceiving the city from a domain-specific point of view. These varied perceptions are also observed at the scale of single buildings, where involved disciplines are much less divergent than at the urban scale. The fundamental differences in common and standard building representation schemas such as IFC [14] and gbXML [15] are rooted in these multiple viewpoints. However, the scale and scope of building related data makes a mapping between these perceptions possible (See, for example, [16]).

At the urban scale, though, the wide range of disciplines and authorities involved in the production and storage of the urban data, results in a large number of parallel perceptions of the urban environment. The available data sources may capture certain aspects of the urban environment and ignore others, view the same physical phenomena from different perspectives, or use different terminologies to express the same concept. A building for instance may be viewed as an active agent from the urban performance point of view, as a mere physical obstruction from the mobility point of view or ignored completely by a botanist.

Analyzing this data to find strategies to overcome various types of problems respond to different inquiries or identify hidden opportunities is hampered not only by the overwhelming size and diversity of the available data, but also by the multiplicity of perceptions and representations, according to which the data is produced.

In order to effectively utilize urban data, it needs to be properly structured and presented in a coherent and interoperable form. The effectiveness and efficiency of Semantic Web technologies to organize and structure web-based building related data has been tested and approved through the SEMERGY experience. However, to upscale this method to the urban level, the above-mentioned particularities of urban data have to be considered.

\subsection{Data Availability and Currentness}

A data-driven approach to decision making, requires substantial data to support implemented analytical methods. Despite the existence of various types of urban data, certain computational methods or optimization tasks may have informational requirements not met by the existing data sources. Furthermore, time is a significant aspect of a large portion of data pertaining to the built environment. Collection of such data occurs in different intervals, resulting in various temporal resolutions. 
Certain computational methods may require resolutions of data unsupported by the existing data collection structures. Facilitation of collection and storage of urban data in required temporal resolutions is fundamental to any data-driven endeavor.

\section{Related Work}

Use of ontologies to capture and structure urban data was discussed in the field of Geographical Information Systems (GIS) by Fonseca and Egenhofer [17] in the late 1990's, leading to the suggestion of Ontology-Driven Geographical Information Systems (ODGIS) as means to share urban knowledge and data [18]. Fonseca and Egenhofer identified "the lack of formal methods to reuse knowledge and data" as a major obstacle hampering the utilization of existing data and knowledge. They support an object-oriented view of the world to represent geographic entities and propose a system-architecture with four main components. These components are data ontologies, the container (domain model), data warehouses and the User Interface. The user selects the desired type of data by browsing the ontologies through the User Interface. The ontology server provides pointers to relevant data warehouses. The container communicates with the data warehouses commanding the extraction of the desired information while maintaining the ontological hierarchies and structure, serving the data to the end user [17]. In a similar attempt, Pundt and Bishr [19] demonstrate the effectiveness of ontologies in enhancement of interoperability is a specific information community in the field of GIS.

Wiegand and Garcia [20], explore the potential of a task-based Semantic Web model to help automate the discovery of web-based data sources, thereby, facilitating the rapid generation of task-appropriate models. They argue that certain tasks such as crisis management activities require the same types of data, regardless of the location of the event. Establishing formal relationships between a type of task and the types of data sources needed for its completion, adds a level of organization to the data, allowing for the rapid retrieval of the desired data, given the intended task and location. In other words, they extend the work of Fonseca and Egenhofer by establishing links between various GIS-related tasks and the types of data stored in different databases, reducing user effort in the development of the model.

Sharing the same perception or committing to the same conceptualization of the object under study is a pre-condition of data sharing and integration. In order to overcome the predicament of parallel perceptions and expressions, in this case of the urban environment concepts, different solutions have been extensively discussed, which are beyond the scope of this paper [21, 22, 23].

Ongoing research conducted by the authors, concerns communication processes of urban entities (for instance buildings, people, and smart infrastructure). Integrating diverse and distributed data sources establishes the need for a modular and extensible data store design. Experiences in building related data handling [24] shows that these requirements are hardly met by common building data collections. To ease the rigid structure of these static data models, other data storage mechanisms are considered (for instance non-relational). By modifying common databases, a generic and scalable 
data store is developed that can hold any kind of data. Relational concepts cannot provide a solution for this issue.

\section{Proposed Framework}

Based on the experience of the SEMERGY project and the above mentioned research activities, the present contribution would suggest a twofold approach to bridge the informational gap between building scale and urban scale performance evaluation requirements. The proposed strategy, on one hand, utilizes ontologies to structure the available urban data repositories with a task-based approach; and on the other hand, addresses the low-level issues of data collection and storage, where the requirements of the task are not met by available data sources.

A globally acknowledged ontology of the urban environment data shared by all involved disciplines and data providers may be an ideal solution to the data accessibility and heterogeneity issues. However, such a solution, given the diversity of the urban data and the variety of domain representations is non-trivial, to say the least. Even if such a comprehensive urban model (encompassing informational requirements and vocabularies of all involved disciplines and sectors) was possible to develop, the complexity of the schema would render its navigation a daunting task.

Definition of a domain, in our case the domain of energy performance of the urban building stock, reduces the scope of the data substantially, however not enough to warrant a manageable degree of complexity. Specifying defined tasks within this domain helps provide a manageable framework for the development of task-based data ontologies, which facilitate automatic creation of task-based models of the desired geographic location or boundary.

Given the multi-disciplinary nature of nearly any urban performance inquiry, such task-based ontologies must not only acquire data pertaining to various fields, but also define a common basis to associate these various data structures with one another to provide data interoperability. The resulting ontology presents a network of Linked Data relevant to the specified task. Different task-ontologies defined in a certain domain include in all likelihood shared data-sources. Given the inter-connected nature of each ontology, such data overlaps establish links between various task-ontologies resulting in the gradual formation of a network of Linked domain Data.

On the other hand, the existing pool of data can be augmented with virtual (calculated) data and/or qualitative information through inclusion of the intermediate or final results of the performance-operation within the task-ontology. This provides a base for exchange of field knowledge through previously formed interoperable (mapable) task-ontologies .

Figure 2 illustrates the proposed ontology structure for two tasks within the same domain. Each task involves two sub-tasks, for accomplishment of which certain data types are required. Each data type is referenced to one (or multiple) datasets belonging to various databases. Based on the geographic location or boundary of the intervention case, the appropriate portion of data from the referenced dataset can be extracted. Different subtasks within a certain task or sub-tasks of different tasks may 
share data requirements (e.g., sub-task 1.2 and 2.1 both utilize dataset B1). If the compatibility issue of the shared dataset with the general schema of one task ontology is solved (for instance through use of a mediator to translate data to the right format), the interoperability of the translated dataset with the second ontology is simultaneously achieved. This diminishes the need for redevelopment of translation methods and mapping schemas for various applications, which share some informational requirements. If various tasks include similar sub-tasks, the results of that specific sub-task linked to one task-ontology can be directly used in the second task (knowledge sharing), provided that the geographic perimeter of the first task includes that of the second.

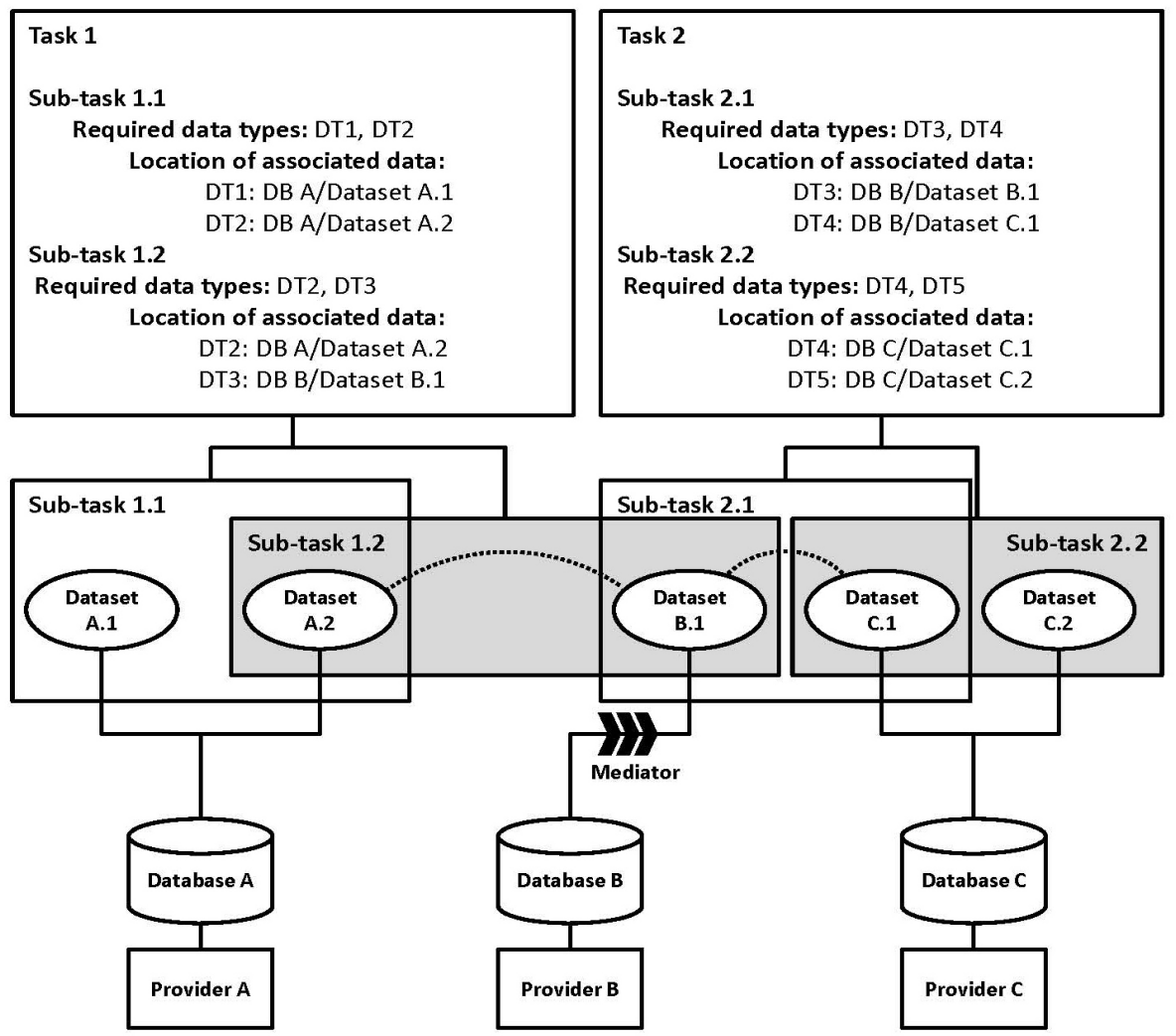

Fig. 2. Proposed task ontology structure

The above schema would help reorganize the existing data to surmount the challenge of web-based data navigation for a specific task. However, data availability issues (within the limits of the task at hand) must be solved before the computational procedures can utilize the data. Each building typically maintains its own data store. These stores handle similar data, however it is not possible to integrate the data in a generic structure. In the majority of cases this is not caused by differences in the data typology but simply by differences in the data storage concepts. Generalizing data 
stores facilitates automated enhancement of data models at runtime, suitable to solve a certain task. In case certain types of data required by the task are not available, the ontology assumes the generic database as the source of data, thereby issuing a request for the collection of the missing data. Collection and storage of unknown urban Big Data can be supported by generic storage concepts. Custom data might be stored in generic databases regardless of the data model. Such databases do not have a fix structure. Rather, the data model is extended at runtime to fit the incoming information. Regarding the importance and size of imports, new nodes might be added on demand.

Requested data types are collected via automated search routines (i.e. web crawl) and made available to the computational procedure through the ontology. Such an approach would support task-based real-time data collection and utilization (Figure 3).

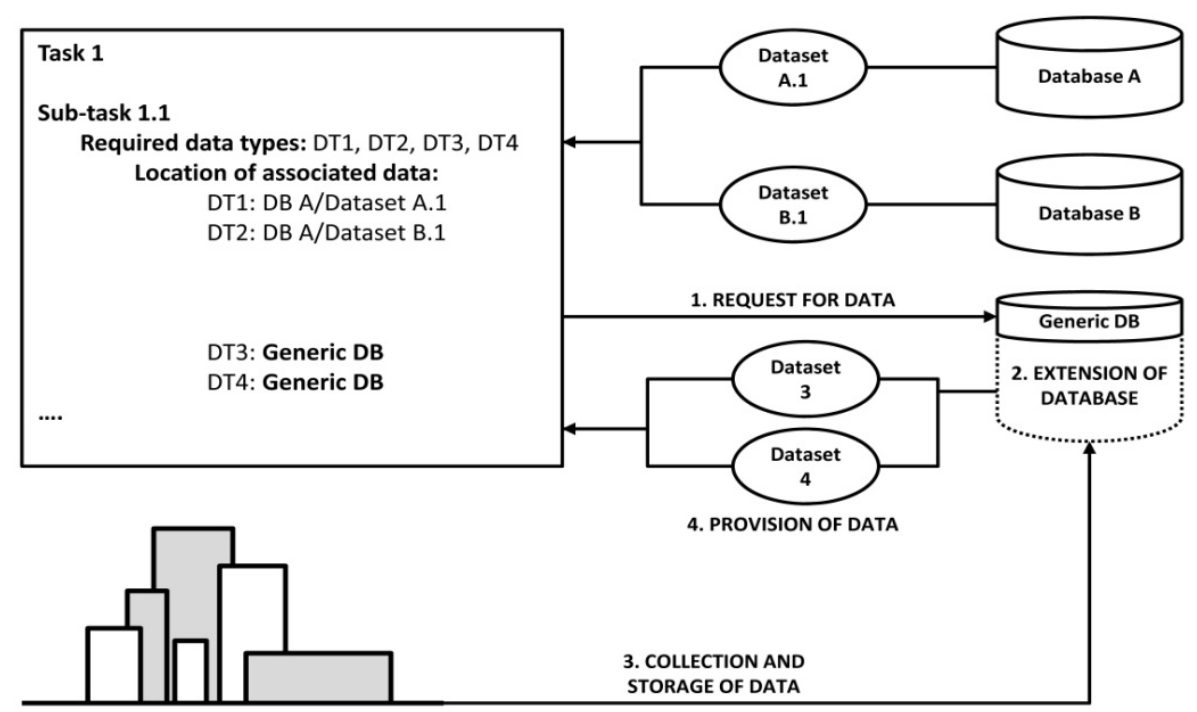

Fig. 3. Collection and provision of required data

In order to explore and demonstrate the potential of the above method to extend the SEMERGY approach to urban scale, the following steps have to be taken.

1. Definition of a task

2. Definition of relative sub-tasks (preferably such that further tasks in the same domain may be supported by the outcomes of these sub-tasks)

3. Identification of required data types

4. Identification of associated existing data-bases and missing data

5. Identification of an ontological representation compliant with the involved datasets and the task in hand (where such a unique structure does not exist, a convenient representation is to be selected in accordance with the data composition and the task nature) 
6. Development of methods or mediators to help represent data from non-compatible sources in the above-mentioned vocabulary and structure

7. Integration of the extensible database as a generic container of the missing data in the developed ontology

8. Automatic readjustment of the generic data structure to the required data

9. Collection and storage of the required yet missing data

10. Integration of computational and visual analytics methods to accomplish the desired sub-tasks (and ultimately task), using the developed task ontology

11. Organization of the intermediate and final results under new ontology classes linked to the original data

12. Development of an appropriate interface to support user interaction

\section{Conclusion}

Sustainable design and retrofit of the built environment requires software solutions to facilitate integration of performance assessment and optimization in the design process. Such software solutions should cater for interventions at building scale, as well as at larger (neighborhood and urban) scales. SEMERGY is a recent research and development effort, aimed at addressing the data-related barriers to performancebased design by exploring the potential of Semantic Web technologies towards utilization of the abundant, yet ill-structured web-based data. Ongoing work intends to extend and augment SEMERGY so as to transcend building scale performance optimization towards urban scale.

Various characteristics of urban data including the overwhelming size, diversity, and multiplicity of perceptions and representations hamper the efficient use of such data in urban performance analysis and optimization tasks. Based on the SEMERGY experience at building scale and previous research on implementation of ontologies (Linked Data concept) for semi-automatic generation of domain models in the field of GIS, a twofold approach was proposed to overcome the data navigation challenge. A task-based approach to creating domain ontologies reduces the scope of the data to a manageable size. Moreover, such an approach provides a strong basis for exchange of previously structured data and knowledge derived from related tasks, within a shared geographical boundary.

Despite the importance of organizing the available yet unstructured data, such a top-down approach is not sufficient. The process of data collection and storage should be supported by generic, efficient, and sizable data structures to tackle the low-level urban data issues. Integration of such databases in the task-based ontology facilitates the task-based automatic retrieval of the missing data for computational purposes.

Once such a data structure is in place, various intervention scenarios can be simulated and optimization techniques may be employed to identify the best course of action in response to objectives pertaining to energy efficiency, environmental performance, and spatial quality of urban domains. Such a data-driven approach could alter conventional problem-solving trends, actively engage stakeholders, accelerate change, and support realization of long term goals. 
Acknowledgements. The SEMERGY project (first phase) was funded under the FFG Research Studio Austrian Program (grant No. 832012) by the Austrian Federal Ministry of Economy, Family and Youth (BMWFJ). In addition to the authors, the SEMERGY team includes: A. M. Tjoa, F. Shayeganfar, A. Anjomshoaa, S. Fenz, J. Heurix, T. Neubauer, C. Sustr, M. Taheri, D. Wolosiuk, K. Hammerberg, and A. Wurm.

\section{References}

1. BSRIA Topic Guides- Building Performance Evaluation. BSRIA Limited, Berkshire (2011)

2. Hensen, J., Djunaedy, E., Radosevic, M., Yahiaoui, A.: Building Performance Simulation for Better Design: Some Issues and Solutions. In: de Wit, M.H. (ed.) Proceedings of the 21th Conference on Passive and Low Energy Architecture, vol. 2, pp. 1185-1190. Eindhoven University of Technology, Eindhoven (2004)

3. Pang, X., Hong, T., Piette, M.A.: Improving Building Performance at Urban Scale with a Framework for Real-Time Data Sharing. Report. Ernest Orlando Lawrence Berkeley National Laboratory, San Diego (2013)

4. Alsaadani, S., Bleil De Souza, C.: The Social Component of Building Performance Simulation- Understanding Architects. In: Wright, J., Cook, M. (eds.) Proceedings of the 2012 Building Simulation and Optimization Conference, Loughborough, pp. 332-339 (2012)

5. Attia, S.: State of the Art of Existing Early Design Simulation Tools for Net Zero Energy Buildings: a Comparison of Ten Tools. Université catholique de Louvain, Louvain La Neuve (2011)

6. Mahdavi, A., El-Bellahy, S.: Efforts and Effectiveness Considerations in Computational Design Evaluation: a Case Study. Building and Environment 40(12), 1651-1664 (2005)

7. Mahdavi, A., Pont, U., Shayeganfar, F., Ghiassi, N., Anjomshoaa, A., Fenz, S., Heurix, J., Neubauer, T., Tjoa, A.: SEMERGY: Semantic Web Technology Support for Comprehensive Building Design Assessment. In: Gudnason, G., Scherer, R. (eds.) eWork and eBusiness in Architecture, Engineering and Construction, pp. 363-370. Taylor\&Francis, Reykjaví (2012)

8. Mahdavi, A., Pont, U., Shayeganfar, F., Ghiassi, N., Anjomshoaa, A., Fenz, S., Heurix, J., Neubauer, T., Tjoa, A.M.: Exploring the Utility of Semantic Web Technology in Building Performance Simulation. In: Proceedings of BauSIM 2012, pp. 58-64. Universität der Künste Berlin, Berlin (2012)

9. Ghiassi, N., Shayeganfar, F., Pont, U., Mahdavi, A., Heurix, J., Fenz, S., Anjomshoaa, A., Tjoa, A.M.: A Comprehensive Building Model for Performance-Guided Decision Support. In: Mahdavi, A., Martens, B. (eds.) Proceedings of the 2nd Central European Symposium on Building Physics, pp. 35-42. Vienna University of Technology, Vienna (2013)

10. Ghiassi, N., Shayeganfar, F., Pont, U., Mahdavi, A., Fenz, A., Heurix, A., Anjomshoaa, A., Neubauer, T., Tjoa, A.M.: Improving the Usability of Energy Simulation Applications in Processing Common Building Performance Inquiries. In: Sikula, O., Hirs, J. (eds.) Simulace Budov a Techniky Prostredi. Ceska Technika - nakladatelstvi, Brno (2012)

11. Shayeganfar, F., Anjomshoaa, A., Heurix, J., Sustr, C., Ghiassi, N., Pont, U., Fenz, S., Neubauer, T., Tjoa, A.M., Mahdavi, A.: An Ontology-Aided Optimization Approach to Eco-Efficient Building Design. In: Proceedings of the 13th Conference of International Building Performance Simulation Association, Chambery, pp. 2194-2200 (2013) 
12. Pont, U., Shayeganfar, F., Ghiassi, N., Taheri, M., Sustr, C., Mahdavi, A., Heurix, J., Fenz, S., Anjomshoaa, A., Neubauer, T., Tjoa, A.M.: Recent Advances in SEMERGY: a Semantically Enriched Optimization Environment for Performance-Guided Building Design and Refurbishment. In: Mahdavi, A., Martens, B. (eds.) Proceedings of the 2nd Central European Symposium on Building Physics, pp. 35-42. Vienna University of Technology, Vienna (2013)

13. Heurix, J., Taheri, M., Shayeganfar, F., Fenz, S., Pont, U., Ghiassi, N., Anjomshoaa, A., Sustr, C., Neubauer, T., Mahdavi, A., Tjoa, A.M.: Multi-Objective Optimization in the SEMERGY Environment for Sustainable Building Design and Retrofit. In: Mahdavi, A., Martens, B. (eds.) Proceedings of the 2nd Central European Symposium on Building Physics, pp. 35-42. Vienna University of Technology, Vienna (2013)

14. Building Smart, http://www.buildingsmart-tech.org

15. gbXML, http://www.gbxml. org/aboutgbxml.php

16. Bazjanac, V.: IFC BIM-Based Methodology for Semi-Automated Building Energy Performance Simulation. In: Rischmoller, L. (ed.) Proceedings of the 25th CIB W78 Conference: Improving the Management of Construction Projects Through IT Adoption. Universidad de Talca, Santiago (2008)

17. Fonseca, F.T., Egenhofer, M.J.: Ontology-Driven Geographic Information Systems. In: Bauzer Medeiros, C. (ed.) zth ACM Symposium in Geographic Information Systems, Kansas (1999)

18. Fonseca, F.T., Egenhofer, M.J., Davis Jr., C.A., Borges, K.A.V.: Ontologies and Knowledge Sharing in Urban GIS. Computer, Environment and Urban Systems 24(3), 232-251 (2000)

19. Pundt, H., Bishr, Y.: Domain Ontologies for Data Sharing- an Example from Environmental Monitoring Using Field GIS. In: Computers and Geosciences, vol. 28, pp. 95-102. Elsevier (2002)

20. Wiegand, N., Garcia, C.: A Task-Based Ontology Approach to Automate Geospatial Data Retrieval. Transactions in GIS 11(3), 335-376 (2007)

21. Bergamaschi, S., Castano, S., De Capitani di Vimercati, S., Montanari, S., Vincini, M.: An Intelligent Approach to Information Integration. In: Guarino, N. (ed.) Formal Ontology in Information Systems. IOS Press, Amsterdam (1998)

22. Rodríguez, M.A., Egenhofer, M., Rugg, R.D.: Assessing Semantic Similarities among Geospatial Feature Class Definitions. In: Včkovski, A., Brassel, K.E., Schek, H.-J. (eds.) INTEROP 1999. LNCS, vol. 1580, pp. 189-202. Springer, Heidelberg (1999)

23. Bishr, Y.: Overcoming the Semantic and other Barriers to GIS Interoperability. International Journal of Geographic Information Science 12(4), 299-314 (1998)

24. Zach, R., Schuss, M., Braeuer, R., Mahdavi, A.: Improving Building Monitoring Using a Data Preprocessing Storage Engine Based on Mysql. In: Gudnason, G., Scherer, R., et al. (eds.) eWork and eBusiness in Architecture, Engineering and Construction, pp. 151-157. Taylor \& Francis (2012) 